\title{
TIME FOR THE NEXT PHASE OF STROKE RECOVERY TRIALS
}

KEY WORDS: Stroke, Recovery, Fluoxetine, Stroke, Disability, Depression,

Number of words: 1060

Authors: Kwakkel Ga,b, Meskers CGM ${ }^{a, b}$, Ward NS ${ }^{c, d}$

a Department of Rehabilitation Medicine, Amsterdam UMC, Vrije Universiteit Amsterdam, Amsterdam Movement Sciences, Amsterdam Neuroscience, de Boelelaan 1117, Amsterdam, The Netherlands.

b Department of Physical Therapy and Human Movement Sciences, Feinberg School of Medicine, Northwestern University, Chicago, II, United states.

c Department of Clinical and Motor Neuroscience, UCL Queen Square Institute of Neurology, Queen Square, London WC1N 3BG, UK.

d The National Hospital for Neurology and Neurosurgery, Queen Square, London WC1N 3BG, UK.

Nine years ago, the FLAME trial ${ }^{1}$ suggested that early prescription of daily $20 \mathrm{mg}$ fluoxetine, a selective serotonin reuptake Inhibitor (SSRI), could dramatically enhance upper and lower limb motor recovery measured with the Fugl-Meyer motor scale in patients with moderate to severe ischemic stroke. The rationale for using fluoxetine was that it would enhance spontaneous post-stroke plasticity mechanisms with beneficial effects on functional recovery ${ }^{1}$. FLAME put fluoxetine in the spotlight as a 'recovery' drug but it was a relatively small trial $(\mathrm{N}=118)$ and did not generate sufficient evidence on its own to change practice ${ }^{1}$.

In the present issue of the Lancet Neurology, two almost identical high-quality phase III trials, the AFFINITY ${ }^{2}$ and EFFECTS ${ }^{3}$ trials, examined the effects of fluoxetine on disability 6 months after stroke. The AFFINITY trial is an international double-blind, placebo-controlled trial conducted in Australia, New Zealand and Vietnam ( $N=1280)$, whereas the EFFECTS trial is a national trial conducted at 35 sites $(\mathrm{N}=1500)$ in Sweden. (Table 1) These two trials followed the larger FOCUS trial conducted at 103 sites in the UK $(n=3127)^{4}$. All three trials convincingly show that the use of $20 \mathrm{mg}$ fluoxetine prescribed daily and starting 2-15 days after stroke has no effects on the modified Rankin Scale (mRS), as illustrated by an identical common odds for the AFFINITY $(0.94,95 \% \mathrm{Cl}: 0.76-1.15 ; \mathrm{p}=0.53)^{2}$, EFFECTS trial $(0.94,95 \% \mathrm{Cl}: 0.78-1.13 ; \mathrm{p}=0.42)^{3}$, and FOCUS $(0.95,95 \% \mathrm{Cl}: 0.84-1.08 ; p=044)^{4}$. As expected, there were beneficial effects on mood and emotional control in both trials ${ }^{2,3}$ with a significant lower proportion of new depressions in the EFFECTS trial ${ }^{3}$, it is also clear that daily use of fluoxetine may also cause harm with an increased incidence in number of serious falls ${ }^{2}$, with more bone fractures of 2$3 \%^{2,3}$, epileptic seizures ${ }^{2}$, hyponatremia ${ }^{3}$ and uncontrolled diabetes ${ }^{3}$ in the first 6 months post stroke. So, the promising results from the FLAME trial ${ }^{1}$ have not been supported. The AFFINITY2 ${ }^{2}$ EFFECTS ${ }^{3}$ and FOCUS ${ }^{4}$ trials (combined $\mathrm{N}=5907$ ) had high adherence and a low risk of bias, so we now have further class I evidence that the simple daily prescription of $20 \mathrm{mg}$ fluoxetine cannot be recommended for patients with a mild ${ }^{3}$ to moderate ${ }^{2}$ stroke undergoing routine post-stroke care. The differences and similarities between the results of all four trials are shown in Supplementary Table 1.

Before closing the chapter on pharmacological enhancement of recovery after brain injury however, it is worth revisiting why fluoxetine was considered a recovery drug in the first place. 
In fact, there is a wealth of evidence from animal models that fluoxetine, and SSRIs in general, can increase the potential for activity-dependent plasticity in the adult brain, most likely through a reduction of extracellular levels of GABA and an increase in BDNF-expression ${ }^{5}$. There has been interest in the modulation of other neurotransmitter systems to promote recovery after brain injury but it has always been understood that drugs help only when combined with appropriate environmental experience ${ }^{6}$. This crucial difference between drugs as enhancers of physical or behavioural training effects rather than restorative agents was not appreciated in the design of the FOCUS, AFFINITY and EFFECTS trials. There was no attempt to influence or even measure the physical or behavioural training so important for stroke recovery. It has been suggested ${ }^{7}$ that the identical outcomes used in the FOCUS, AFFINITY and EFFECTS trials will allow for a combined secondary analysis to look for interactions with the amount of exercise therapy provided at an individual level. However, the dose of usual care rehabilitation is likely insufficient in all three trials for this to be revealing. ${ }^{6,8}$ The key clinical question, whether pharmacotherapy can modulate an adequate dose of physical or behavioral training to enhance stroke recovery, has yet to be addressed in a phase III trial.

Another contentious area is that of appropriate outcome measures. FOCUS ${ }^{4}$, AFFINITY $^{2}$ and EFFECTS ${ }^{3}$ used the $\mathrm{mRS}$, the most commonly used outcome score in large stroke recovery trials, as the primary outcome score. In the FLAME trial ${ }^{1}$, the mRS (a secondary outcome measure) showed a significantly greater improvement in the fluoxetine group, and so attempting to replicate this result further in the larger trials is understandable. However, given its likely mechanism of action, fluoxetine is most likely to impact at the level of impairment and so attempting to replicate the effect of fluoxetine on impairment seen in $\mathrm{FLAME}^{1}$ would have been useful. Changes in impairment do not necessarily universally translate into reduced overall disability assessed by mRS. With this in mind, the recent Stroke Recovery and Rehabilitation Roundtable (SRRR) group published several consensus documents on how to harmonize stroke recovery research with respect to stratified trial design, using biomarkers and outcome measures ${ }^{8}$. With a shared vision, the SRRR group recommended a set of additional validated performance tests, beyond the use of the mRS for stroke recovery and rehabilitation trials ${ }^{9}$. One hope is that careful selection of primary and secondary outcome measures might allow characterization of those people more likely to respond in different ways (reduced impairment, improved activity and/or participation), leading to further targeted trials with appropriate stratification ${ }^{10}$. In the case of recovery of behavior for example, there is an urgent need to measure quality of movement in stroke recovery and rehabilitation trials to understand what patients learn and how they improve their motor performance after stroke ${ }^{9}$. We note that several organisations have adopted these recommendations as important considerations when appraising funding applications.

The current three almost identical trials arrived together. Whilst we acknowledge that AFFINITY ${ }^{2}$ and EFFECTS ${ }^{3}$ would have been started before FOCUS ${ }^{4}$ concluded, it is now not clear what their added value is. It seems that the same lemon is squeezed not once, but twice more with necessarily diminishing returns. In a world of limited resources, it illustrates the need for a global agenda for stroke recovery research allowing the optimal alignment of trials to address complementary research questions. To improve stroke recovery research, we foresee a growing need for the role of SRRR in designing and delivering improved and more robust translational trials that bridge the gap between pre-clinical and clinical fields. In particular, there is an important role for the Global Alliance of Independent Networks focused on stroke 
Trials (GAINS) (https://www.globalstroketrials.org/) to improve communication, alignment and complementary (not identical) design of phase III and IV trials between the excellent national networks that already exist worldwide in stroke research. We hope this does not signal the end of pharmacotherapy for stroke recovery, but rather the beginning of the next phase.

\section{REFERENCES}

1. Chollet F, Tardy J, Albucher JF, Thalamas C, Berard E, Lamy C, Bejot Y, Deltour S, Jaillard A, Niclot P, Guillon B, Moulin T, Marque P, Pariente J, Arnaud C, Loubinoux I. Fluoxetine for motor recovery after acute ischaemic stroke (FLAME): a randomised placebo-controlled trial. Lancet Neurol. 2011 Feb;10(2):123-30. doi: 10.1016/S1474-4422(10)70314-8. Epub 2011 Jan 7. Erratum in: Lancet Neurol. 2011 Mar;10(3):205. PubMed PMID: 21216670.

2. AFFINITY trial Collaboration. Safety and efficacy of fluoxetine on functional outcome after acute stroke (AFFINITY): a randomised, double-blind, placebo-controlled trial. Lancet Neurology (in press)

3. EFFECTS trial collaboration. EFFECTS Efficacy of fluoxetine - a randomised controlled trial in stroke (EFFECTS). Lancet Neurology (in press)

4. FOCUS Trial Collaboration. Effects of fluoxetine on functional outcomes after acute stroke (FOCUS): a pragmatic, double-blind, randomised, controlled trial. Lancet. 2019 Jan 19;393(10168):265-274. doi: 10.1016/S0140-6736(18)32823-X. Epub 2018 Dec 5. PubMed PMID: 30528472; PubMed Central PMCID: PMC6336936.

5. Maya Vetencourt JF, Sale A, Viegi A, Baroncelli L, De Pasquale R, O'Leary OF, Castrén E, Maffei $L$. The antidepressant fluoxetine restores plasticity in the adult visual cortex. Science. 2008 Apr 18;320(5874):385-8. doi: 10.1126/science.1150516. PubMed PMID: 18420937.

6. Ward NS. Restoring brain function after stroke - bridging the gap between animals and humans. Nat Rev Neurol. 2017 Apr;13(4):244-255. doi: 10.1038/nrneurol.2017.34. Epub 2017 Mar 17. Review. PubMed PMID: 28303914.

7. Mead GE, Legg L, Tilney R, Hsieh CF, Wu S, Lundström E, Rudberg AS, Kutlubaev M, Dennis MS, Soleimani B, Barugh A, Hackett ML, Hankey GJ. Fluoxetine for stroke recovery: Metaanalysis of randomized controlled trials. Int J Stroke. 2019 Oct 17:1747493019879655. doi: 10.1177/1747493019879655. [Epub ahead of print] PubMed PMID: 31619137.

8. Bernhardt J, Borschmann K, Boyd L, Carmichael ST, Corbett D, Cramer SC, Hoffmann T, Kwakkel G, Savitz S, Saposnik G, Walker M, Ward N. Moving Rehabilitation Research Forward: Developing Consensus Statements for Rehabilitation and Recovery Research. Neurorehabil Neural Repair. 2017 Aug;31(8):694-698. doi: 10.1177/1545968317724290. PubMed PMID: 28803534.

9. Kwakkel G, Lannin NA, Borschmann K, English C, Ali M, Churilov L, Saposnik G, Winstein C, van Wegen EEH, Wolf SL, Krakauer JW, Bernhardt J. Standardized Measurement of 
Sensorimotor Recovery in Stroke Trials: Consensus-Based Core Recommendations from the Stroke Recovery and Rehabilitation Roundtable. Neurorehabil Neural Repair. 2017 Sep;31(9):784-792. doi: 10.1177/1545968317732662. PubMed PMID: 28934918.

10. van der Vliet R, Selles RW, Andrinopoulou ER, Nijland R, Ribbers GM, Frens MA, Meskers C, Kwakkel G. Predicting Upper Limb Motor Impairment Recovery after Stroke: A Mixture Model. Ann Neurol. 2020 Mar;87(3):383-393. doi: 10.1002/ana.25679. Epub 2020 Jan 25. PubMed PMID: 31925838; PubMed Central PMCID: PMC7065018. 